\title{
Se réinventer pour former les étudiants en situation de pandémie mondiale - le cas d'une enseignante en technique d'hygiène dentaire
}

\author{
Auteures \\ Catherine Vachon, doctorante en éducation, Université du Québec à \\ Chicoutimi, Canada, \\ catherine.vachon1@uqac.ca
}

Hélène Charest, enseignante en technique d'hygiène dentaire, cégep de Chicoutimi, Canada,

hcharest@cchic.ca 


\section{REVUE HYBRIDE DE L’ÉDUCATION}

\section{Résumé}

La pandémie mondiale liée à la COVID-19 a provoqué des changements sans précédent dans le monde entier, notamment en éducation. Lors de la déclaration de l'état d'urgence sanitaire en mars 2020, des centaines d'enseignants provenant de partout au Québec ont dû modifier leurs stratégies d'enseignement rapidement afin que les apprenants aient accès à l'éducation à laquelle ils ont droit. Ces besoins d'adaptation ont ainsi amené madame Hélène Charest, enseignante en technique d'hygiène dentaire au cégep de Chicoutimi, à travailler avec ses étudiants pour créer du matériel de prévention, contribuant ainsi à la formation obligatoire des étudiants en technique d'hygiène dentaire et à l'offre de services aux enfants fréquentant des écoles et des centres de la petite enfance de la région du Saguenay-Lac-Saint-Jean.

Mots-clés : hygiène dentaire; enseignement au collégial; adaptation de l'enseignement; outils technologiques; COVID-19; pandémie mondiale 


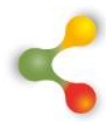

\section{REVUE HYBRIDE DE L'ÉDUCATION}

\section{Mise en contexte}

Le 13 mars 2020, le gouvernement du Québec a déclaré l'état d'urgence sanitaire, forçant les citoyens à vivre une série d'évènements sans précédent (ministère de la Santé et des Services sociaux, 2020). Cette situation a mené au confinement de la population, impliquant ainsi la fermeture de nombreux commerces et de nombreux services essentiels tels que les établissements scolaires, qu'il s'agisse des services publics ou privés, et ce, autant pour les jeunes que pour les adultes. Cette fermeture a amené de grands changements, notamment la création et l'utilisation de plateformes numériques visant à soutenir les apprentissages et obligeant les enseignants à modifier leurs stratégies d'enseignement en utilisant des ressources numériques afin d'enseigner à distance (ministère de l'Éducation et de l'Enseignement supérieur, 2020).

Le domaine de l'éducation est un domaine sollicitant particulièrement la capacité d'adaptation des individus, que ce soit de la part des apprenants, des enseignants, des parents ou de tout autres acteurs du milieu scolaire. Les réformes scolaires, les changements sociétaux, les nouvelles modalités d'enseignement et les besoins en perpétuel changement du monde du travail constituent des vecteurs de changement dans les institutions d'enseignement, et ce, partout dans le monde, amenant les enseignants à devenir créatifs et prêts à se renouveler.

Les enseignants, peu importe le niveau scolaire auquel ils enseignent, se sont adaptés aux besoins des apprenants et des milieux de pratique, ainsi qu'aux contraintes qui s'imposent. Celles-ci pouvaient être, par exemple, liées au contexte de formation ou aux exigences des programmes de formation. Cependant, en 2020, les enseignants ont dû faire preuve d'une grande capacité d'adaptation et d'ingéniosité pour surmonter les embûches liées au contexte de pandémie mondiale qui a grandement modifié les contextes d'apprentissages. Les bouleversements causés par cette crise sanitaire ont été nombreux: fermeture des établissements d'enseignement, interdiction de partager du matériel, pénurie de nombreux produits, nouvelles modalités d'enseignement, nouvelles contraintes de travail dans les écoles, etc.

Plusieurs enseignants se sont retrouvés devant de nouveaux problèmes, notamment l'impossibilité d'enseigner en présentiel, de donner la chance aux apprenants de faire des stages en milieu de pratique ou alors de leur faire vivre les activités prévues en classe. Au niveau collégial, ce sont des centaines d'étudiants qui se sont retrouvés isolés, sans possibilité d'aller en classe et sans accès à du matériel, et de nombreux enseignants qui ont dû réapprendre à enseigner en utilisant la technologie pour communiquer à distance. C'est le cas de madame Hélène Charest, enseignante en technique d'hygiène dentaire au cégep de Chicoutimi. 


\section{8}

\section{REVUE HYBRIDE DE L'ÉDUCATION}

Madame Charest enseigne depuis 2011 au cégep de Chicoutimi. Détentrice d'un diplôme d'études supérieures spécialisées (DESS) en enseignement au collégial, elle poursuit sa formation afin de pouvoir offrir un enseignement de qualité à ses étudiants. Elle termine en ce moment une maîtrise jumelée à des formations sur l'enseignement en ligne qu'elle a débutées pour répondre aux besoins des apprenants dans ce contexte de pandémie.

Enseignante à l'écoute et qui s'adapte aux besoins de ses étudiants, madame Charest insiste sur le fait que les apprenants doivent s'exprimer et manifester leur inconfort ou leurs difficultés afin d'y trouver des solutions. Cela est particulièrement vrai dans un contexte de formation à distance puisqu'il demeure difficile, voire parfois impossible, d'observer ses apprenants. Elle a su faire preuve de créativité afin d'adapter ses stratégies d'enseignement au contexte dans lequel doivent évoluer ses étudiants en temps de pandémie. Cet article se veut donc une présentation de son projet d'enseignement qui a permis à ses étudiants de se dépasser et de se développer professionnellement malgré les contraintes sanitaires imposées par la pandémie de la COVID-19.

\section{Déroulement}

\section{Quand la pandémie impose un renouveau des stratégies d'enseignement}

Madame Charest enseigne principalement la santé dentaire publique, en plus de superviser les stages dans ce domaine. Généralement, ce cours implique que les étudiants développent des connaissances théoriques, telles que les normes de santé dentaire publique et les obligations et le rôle du technicien en hygiène dentaire œuvrant dans le domaine de la santé publique, mais également des connaissances pratiques, comme l'élaboration d'un plan d'accompagnement d'enfants d'âge scolaire bénéficiant de soins dentaires à l'école ou l'élaboration de stratégies de prévention des problèmes de santé bucco-dentaire. À l'hiver 2020, madame Charest avait donc planifié des périodes d'enseignement magistral, des moments de partage et de coconstruction des connaissances entre étudiants et des travaux réalisés en laboratoire et lors des stages. Cependant, l'arrivée de la COVID-19 a chamboulé ses plans, l'obligeant à s'adapter.

Lorsque le premier confinement a été imposé, l'ensemble des établissements scolaires ont fermé, tout comme une majorité de commerces et de services. Madame Charest, qui devait accompagner ses étudiants en stage, s'est vue prise au dépourvu, l'obligeant à modifier drastiquement sa planification puisque les stagiaires ne pouvaient plus aller dans les écoles où ils devaient réaliser leurs apprentissages pratiques. Si certains stagiaires avaient été en mesure de compléter leur stage peu avant le confinement, d'autres devaient y accorder encore de nombreuses 


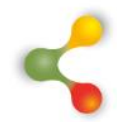

\section{REVUE HYBRIDE DE L'ÉDUCATION}

heures pour compléter leur formation. Pour répondre aux besoins des étudiants et aux exigences de formation, ainsi que pour offrir à ses étudiants une expérience de formation enrichissante, madame Charest a contacté chacun de ses 25 étudiants dans le but de leur créer un plan de formation individualisé en fonction des objectifs à atteindre et du respect des exigences du programme. Ces rencontres lui ont donc permis d'en savoir plus sur les besoins de ses étudiants, notamment en ce qui a trait au besoin de matériel leur permettant de poursuivre leur formation, mais aussi de les rassurer. Ces rencontres lui ont aussi permis de s'informer sur les besoins de ces étudiants en ce qui a trait aux adaptations de ses méthodes d'enseignement (ouverture des étudiants, disponibilité, accessibilité au matériel informatique, etc.). Madame Charest était consciente que l'apprentissage à la maison représentait un défi pour de nombreux étudiants et que c'était son devoir de s'assurer que tous puissent se développer et profiter d'un enseignement de qualité qui répond à leurs besoins.

C'est donc à la suite de ces rencontres que les étudiants en technique d'hygiène dentaire ont pu bénéficier d'un enseignement adapté. Puisqu'il était impossible pour eux de faire des stages, l'enseignante a créé des simulations et des études de cas qui représentaient des aspects réels de la profession que les étudiants seraient susceptibles de rencontrer au cours de leur carrière. Bien que ce changement de méthode d'apprentissage ait permis aux étudiants de développer les connaissances et les compétences nécessaires à leur entrée sur le marché du travail, madame Charest savait que cela ne serait pas tout à fait complet et désirait modifier une fois de plus sa stratégie d'enseignement pour les étudiants de l'année suivante qui devraient eux aussi faire des stages dans des conditions de distanciation sociale liées à la pandémie.

\section{Savoir se réinventer pour atteindre les exigences}

Madame Charest a donc décidé de prendre les choses en main. Curieuse et désireuse d'offrir un enseignement adapté, elle s'est tournée vers les formations à distance afin de développer ses connaissances en technologie, ce qui lui permettait de se servir plus aisément de logiciels et d'outils d'enseignement à distance. Cette ouverture à la technologie l'a amenée à réfléchir à la possibilité d'utiliser celle-ci avec les étudiants afin que ces derniers puissent vivre leur stage différemment.

La formation de futurs professionnels exige le respect d'exigences et d'obligations liées aux différentes connaissances que doivent développer les étudiants, ainsi qu'au nombre d'heures que certains étudiants doivent réserver à la pratique d'actes professionnels supervisés. La formation offerte aux futurs techniciens en santé dentaire n'y fait pas exception. Madame Charest devait donc s'assurer de respecter les curriculums de formation imposés pour la formation initiale en santé dentaire. 


\section{$\propto$ \\ REVUE HYBRIDE DE L'ÉDUCATION}

Les exigences du programme sont claires : les étudiants doivent être en mesure de produire des programmes de prévention en santé publique en plus d'intervenir dans les écoles afin d'identifier les élèves à risque et de procéder à des actes liés à la profession tels que la pose de scellant. Madame Charest devait donc affronter ce défi. Il était nécessaire d'adapter les stratégies d'enseignement afin que les étudiants puissent atteindre ces objectifs. Ainsi, en considérant la possibilité que les étudiants ne puissent pas aller dans les écoles, l'enseignante a décidé de prendre un virage numérique. En ayant en tête son intention éducative, qui était d'amener les étudiants à être en mesure d'intervenir en santé dentaire, à faire de la prévention en concevant du matériel pédagogique pour une clientèle cible ainsi qu'à connaitre le programme de santé publique, elle a imaginé un projet d'envergure dans lequel les étudiants devraient créer des capsules vidéo ayant pour but de prévenir les problèmes de santé dentaire chez les jeunes. II s'agissait donc d'une toute nouvelle forme de développement des connaissances professionnelles chez les étudiants, mais qui permettait d'atteindre toutes les exigences et obligations de la formation des techniciens en santé dentaire. Ces capsules étaient accompagnées d'activités et de matériel éducatifs qui permettaient aux enfants d'acquérir des connaissances et de les démontrer lors de la réalisation d'activités de réinvestissement. Madame Charest désirait aussi que les étudiants créent des boites contenant du matériel pédagogique et des directives élaborées par les étudiants qui permettraient à ces derniers d'évaluer si leur programme de prévention, jumelé aux capsules vidéo, permettait aux enfants d'acquérir des apprentissages, et ce, grâce à la collaboration des enseignants et des éducateurs œuvrant dans les centres de la petite enfance.

L'élaboration d'une telle stratégie d'enseignement exige non seulement du nouveau matériel, mais également certaines compétences en lien avec la technologie ainsi que la collaboration de plusieurs personnes. En effet, la création de capsules vidéo nécessite d'avoir accès à des ordinateurs, des caméras et des logiciels de montage. Cela implique également une certaine habileté pour l'utilisation des programmes informatiques qui ne sont pas d'emblée enseignés dans la formation des techniciens en hygiène dentaire. II fallait d'abord s'assurer que les étudiants soient en accord avec ce projet qui n'avait jamais été fait auparavant, qu'ils soient à l'aise de participer à la création de vidéos et qu'ils aient tous accès au matériel nécessaire. Cela était un point particulièrement important puisque, comme le mentionne l'enseignante :

Certains étudiants ne possédaient qu'un cellulaire ou une tablette et venaient faire leurs travaux au collège. Le simple fait d'être en confinement compliquait la réalisation des travaux habituels. Je devais vraiment trouver un moyen pour que tous aient accès à un ordinateur. 


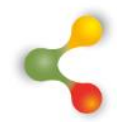

\section{REVUE HYBRIDE DE L'ÉDUCATION}

Ainsi, avant même de présenter l'idée aux étudiants, il s'est avéré nécessaire de communiquer avec de nombreux collègues que ce soit des membres de la direction, des techniciens en informatique, des conseillers pédagogiques et des responsables de prêts de matériel technologique. Les démarches de madame Charest lui ont permis d'obtenir des prêts d'ordinateurs pour ses étudiants, d'être conseillée pour l'utilisation de logiciels gratuits qui lui permettraient de mener son activité tout en répondant à ses attentes et d'être accompagnée dans l'apprentissage des méthodes d'utilisation de ces nouveaux outils. Madame Charest s'explique :

Ce n'était pas juste de prévoir un apprentissage pour mes étudiants, mais aussi pour moi. Je devais savoir comment utiliser les outils pour pouvoir dépanner mes étudiants en cas de problème. Ça a donc été une grande charge de travail de tout réapprendre, mais je voulais vraiment les aider et je me disais que ces connaissances me seraient utiles l'année suivante.

Ce n'est qu'après ce travail en coulisse qu'elle a été en mesure de mettre sur pieds son projet et de le présenter aux étudiants.

L'enseignante est très claire : il était essentiel que son projet plaise aux étudiants. En effet, pour elle, il était primordial que les étudiants aient envie de s'investir dans un projet comme celui-ci qui nécessite un engagement important. Elle voulait qu'ils se sentent appuyés et prêts, une fois l'activité terminée, à entrer sur le marché du travail. Ce projet devait donc être signifiant pour eux et devait répondre à leurs attentes de formation et d'apprentissage. Elle a donc contacté tous ses étudiants et a abordé l'idée de ce projet avec eux.

Une fois l'accord des étudiants obtenu, il fallait s'assurer de leur fournir les ressources et le soutien nécessaires pour la réalisation de ce projet éducatif. Pour madame Charest, cela nécessitait la collaboration de personnes qui utilisent les outils technologiques impliqués et que ces derniers soient disponibles pour l'aider ou pour aider les étudiants en cas de problème. Un partenariat avec une conseillère en technologie de l'information et de la communication de même qu'avec une technicienne en communications s'est alors créé. Cette association permettait à l'enseignante d'offrir du soutien technique aux étudiants lorsque ceux-ci rencontraient des embûches.

Selon madame Charest, les étudiants ont fait preuve d'une capacité d'adaptation impressionnante, ainsi que d'une motivation spectaculaire. En effet, ceux-ci ont démontré une forte envie de créer du matériel de qualité qui viendrait réellement en aide aux élèves qui y auraient accès. Ces derniers posaient des questions, demandaient de l'aide et participaient aux 


\section{$\&$}

\section{REVUE HYBRIDE DE L'ÉDUCATION}

rencontres avec l'enseignante afin de perfectionner leur matériel et pour se fixer des objectifs spécifiques.

L'adaptation de son enseignement au contexte de pandémie, ainsi que la mise en place de ce projet a, selon madame Charest, modifié son rôle d'enseignante. Celle qui travaille assidûment a dû apprendre à lâcher prise et comprendre que l'on peut atteindre les mêmes objectifs de formation tout en utilisant de nouvelles stratégies d'enseignement. L'enseignante explique que l'importance de la collaboration dans la profession est plus grande que jamais depuis le début de la pandémie et que le fait de travailler avec d'autres professionnels lui a permis de trouver des solutions à de nombreux problèmes.

\section{Apports et prospectives}

\section{Retombées du projet}

La mise en place d'un tel projet implique nécessairement un moment réflexif pendant lequel on se penche sur les retombées et sur les apports dans la pratique et dans le développement professionnel des étudiants. C'est ce qui a amené madame Charest à se questionner sur sa pratique enseignante et sur les conséquences du projet dans la formation de ses étudiants. C'est également cela qui l'a poussée à demander à ses étudiants ce qu'ils ont eux-mêmes vécu par rapport au projet.

Le premier élément que madame Charest a verbalisé est le fait que son projet est allé beaucoup plus vite et plus loin que ce qu'elle pouvait imaginer au départ. En effet, celle qui ne savait pas si le projet allait bien se dérouler a pu constater que les étudiants sont allés au-delà ce qui était souhaité en développant des compétences supplémentaires. Au départ, l'enseignante planifiait ce projet de prévention afin que ses étudiants apprennent à développer des projets d'intervention et de prévention en santé dentaire auprès de jeunes enfants en utilisant les prescriptions des curriculums en matière de santé et d'hygiène dentaire. Or, il s'est avéré que les étudiants ont non seulement atteint cet objectif, mais qu'ils ont également développé des connaissances et des compétences liées à l'utilisation des technologies (ce qui est un apprentissage visé lors de la formation) et de grandes aptitudes liées au travail d'équipe. Les étudiants se sont surpassés, reconnaissant l'expertise et l'aisance de chacun et demandant de l'aide à leurs collègues lorsque le besoin se faisait sentir. De plus, ils ont été en mesure de développer leur capacité à reconnaitre leur force, mais aussi à identifier leurs besoins.

Cette habileté à travailler et à communiquer avec autrui ne s'est pas seulement manifestée auprès de leurs pairs, mais également auprès de professionnels de différents domaines. En effet, madame Charest raconte à quel point les étudiants étaient ouverts et comment ils ont eu la bonne idée de se référer aux professionnels en communication du cégep pour être 


\section{6}

\section{REVUE HYBRIDE DE L'ÉDUCATION}

en mesure de produire des outils de prévention de qualité accessibles au public. De plus, ils ont dû contacter eux-mêmes des professionnels travaillant dans les écoles et dans les centres de la petite enfance afin de leur présenter leur matériel et de leur demander de collaborer avec eux en utilisant leur matériel et en suivant leurs recommandations pour le déroulement des activités créées. Ces compétences n'auraient pas été aussi développées sans ce projet et le contexte particulier dans lequel il a été implanté. C'est pour cette raison que l'enseignante envisage de conserver ce projet pour les années futures, l'amenant à penser à ce qu'elle ferait si elle répétait cette expérience.

\section{Si c'était à refaire}

Lorsque madame Charest a été questionnée sur ce qu'elle changerait si elle devait refaire le projet, elle a mentionné qu'elle ferait le projet de la même manière. En effet, selon elle, le projet, tel qu'il est présentement, a offert de belles opportunités aux étudiants. C'est un projet vivant et accessible qui permet aux étudiants en technique d'hygiène dentaire d'adapter leurs interventions aux besoins actuels et qui, éventuellement, pourrait devenir un atout en offrant de plus grandes possibilités de partage. Elle ajoute cependant que le projet pourrait être enrichi en développant davantage d'outils et de méthodes de partage. Elle mentionne, entre autres, la possibilité de créer des boites d'outils de prévention qui pourraient être acheminées dans les maisons des élèves. Cela permettrait d'offrir à de nombreuses familles des capsules d'information sur l'hygiène dentaire, en plus de mettre à leur disposition du matériel ludique et éducatif qui permettrait aux parents d'intervenir auprès de leurs enfants. L'enseignante explique que ce projet pourrait ouvrir la porte à de toutes nouvelles stratégies d'intervention.

Madame Charest n'exprime aucun regret lorsqu'elle aborde le projet. Elle conseille aux enseignants qui souhaiteraient se lancer dans la réalisation d'un tel projet de s'entourer de professionnels qui les aideront dans cette démarche. Elle propose donc de demander de l'aide aux collèges et aux conseillers pédagogiques qui sont disponibles pour soutenir les enseignants, les outiller en leur offrant des ressources. Ils pourront offrir des formations et du soutien aux étudiants pour l'utilisation des différents logiciels et outils technologiques mis à leur disposition. De plus, elle affirme que c'est grâce au travail d'équipe que l'on peut voir plus loin, envisager d'autres stratégies pour penser en dehors du cadre habituel et ce, que ce soit avec des collègues, des acteurs du milieu ou toute autre personne impliquée. Madame Charest suggère également de s'outiller adéquatement en ayant une tablette électronique pour utiliser des applications spécialisées, un deuxième écran, une tablette graphique ou tout autre outil pouvant faciliter l'enseignement ou le travail à distance.

Cette enseignante suggère également d'être patient et d'apprendre à morceler les étapes de réalisation d'un tel projet afin que celui-ci soit plus 


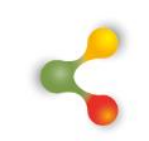

\section{REVUE HYBRIDE DE L'ÉDUCATION}

facile à réaliser. Elle ajoute qu'il faut savoir adapter ses attentes à cette nouvelle réalité et faire preuve de tolérance envers soi-même et envers ses capacités. Elle explique que la mise en place d'un tel projet nécessite une grande flexibilité de la part des enseignants et des milieux de travail et qu'il faut accepter l'erreur qui, selon elle, représente une grande source d'apprentissage et d'amélioration. Madame Charest termine l'entrevue en demandant «qui a dit que tout devait toujours être parfait ? » 


\section{REVUE HYBRIDE DE L’ÉDUCATION}

\section{Références}

Ministère de l'Éducation et de l'Enseignement supérieur. (2020). Coronavirus (COVID-19). Gouvernement du Québec. http://www.education.gouv.qc.ca/coronavirus/

Ministère de la Santé et des Services sociaux. (2020). Coronavirus (COVID-19). Gouvernement du Québec. https://msss.gouv.qc.ca/professionnels/maladiesinfectieuses/coronavirus-2019-ncov/ 